\title{
SUMMING A DIVERGENT SERIES
}

\author{
D. G. C. McKeon
}

It is well known that the manipulation of divergent series can give peculiar results. The series $S(a)=\sum_{n=0}^{\infty} a^{n} n$ ! is clearly horribly divergent for $a \neq 0$, yet we will show by using some elementary integrals involving the Bessel function $K_{0}(x)$ that it can be viewed as an expansion in powers of $a$ of a function which diverges for $a<0$.

The two integrals we need are [1]

$$
\int_{0}^{\infty} d t t^{n} K_{0}(2 \sqrt{t})=\frac{1}{2}(n !)^{2}
$$

and

$$
f(a)=2 \int_{0}^{\infty} d t e^{-a t} K_{0}(2 \sqrt{t})=\frac{e^{\frac{1}{a}}}{a} \int_{1 / a}^{\infty} d t \frac{e^{-t}}{t}(a>0) .
$$

Let us consider expanding $e^{-a t}$ in the argument of (2) so that

$$
f(a)=2 \int_{0}^{\infty} d t \sum_{n=0}^{\infty} \frac{(-a)^{n}}{n !} t^{n} K_{0}(2 \sqrt{t})
$$

integration of this term by term using (1) yields

$$
\begin{aligned}
f(a) & =2 \sum_{n=0}^{\infty} \frac{(-a)^{n}}{n !}\left[\frac{1}{2}(n !)^{2}\right] \\
& =\sum_{n=0}^{\infty}(-a)^{n} n !
\end{aligned}
$$


Thus the divergent series $S(-a)$ can be considered as a representation of the function $f(a)$, which from its definition in $(2)$, is clearly singular for $a<0$ ! (We note that $f(a) \rightarrow 1$ as $a \rightarrow 0^{+}$in both (2) and (4).) This may alternatively be viewed as a way of inserting a "convergence" factor of $1 /(n !)^{2}$ into each term of the series for $S(-a)$.

These manipulations are akin to the insertion of a factor of $\frac{1}{n !}$ in Borel series. To see this, consider the integrals

$$
\int_{0}^{\infty} d t t^{n} e^{-t}=n !
$$

and

$$
g(a)=\int_{0}^{\infty} d t e^{-a t} e^{-t}=\frac{1}{1+a}(a>-1) .
$$

Expanding $e^{-a t}$ in powers of at in (6) we obtain

$$
g(a)=\int_{0}^{\infty} d t \sum_{n=0}^{\infty} \frac{(-a t)^{n}}{n !} e^{-t}
$$

which becomes, if we integrate term-by-term using (5)

$$
\begin{aligned}
g(a) & =\sum_{n=0}^{\infty} \frac{(-a)^{n}}{n !}(n !) \\
& =\sum_{n=0}^{\infty}(-a)^{n} .
\end{aligned}
$$

By comparing (6) with (8), we see that $\frac{1}{1+a}$ is formally represented by the series $\sum_{n=0}^{\infty}(-a)^{n}$ for all $a>-1$ even though this geometric series diverges for $|a|>1$.

We hence see that, once again, a divergent series which at first glance is "meaningless" may in fact be given a "meaning", 
provided we are willing to indulge in interchanging the order of summation and integration, as we have done in going from (2) to (4) or (6) to (8). (As $\sum_{n=0}^{\infty} \frac{(-a t)^{n}}{n !}$ uniformly converges to $e^{-a t}$ for finite at, term-by-term integration of the series in (3) and (7) is justified over any finite range.)

A more formal approach that is clearly related to the usual discussion of Borel Summation is now sketched. If one has a series

$$
f(a)=\sum_{n=0}^{\infty} b_{n} a^{n} n !
$$

which converges for $|a|<R$, then

$$
\phi(a)=\sum_{n=0}^{\infty} \frac{b_{n} a^{n}}{n !}
$$

converges everywhere. It is easily shown now, using the integral of eq. (1), that

$$
F(a)=2 \int_{0}^{\infty} d t \phi(t a) K_{0}(2 \sqrt{t})
$$

is an analytic continuation of $f(a)$ across $|a|=R$ wherever $F(a)$ is analytic. We have essentially considered an example of this general result for the case $b_{n}=(-1)^{n}$ and $R=0$ where the usual arguments are invalid.

\section{References}

[1] I. Gradsteyn and M. Ryzhik, Table of Integrals, Series and Products, 5th ed. Academic Press.

D. C. G. McKeon

Department of Applied Mathematics

University of Western Ontario

London

Canada N6A 5B7 